\title{
The Genetics Corner: Avoiding the Need for Cardiac Transplant - Everolimus Induced Regression of a Cardiac Tumor in an Infant with Tuberous Sclerosis Complex (TSC) Type 2 with Updates on his Progress
}

June-Anne Gold MD

\section{Case Summary}

The patient was prenatally diagnosed with a cardiac tumor; this was attached to the mitral valve. I had suggested during the pregnancy that Everolimus or Sirolimus (mTOR inhibitors) could be given to the mother to reduce the tumor in the fetus. This was only reported in one case previously (1). Parents had declined amniocentesis, so we did not have a molecularly confirmed diagnosis at that time. Cardiac rhabdomyomas are commonly associated with tuberous sclerosis complex types 1 and 2 . This is due to a molecular pathogenic variant in either TSC1 or TSC2. TSC1 and TSC2 are in the mTOR pathway, which would lend itself to treatment with the mTOR inhibitors. These fetal cardiac rhabdomyomas usually spontaneously regress during the pregnancy or immediately after birth and do not cause hemodynamic problems. However, larger tumors can cause an obstruction, as in this case, and intervention is then needed.

\section{"These fetal cardiac rhabdomyomas} usually spontaneously regress during the pregnancy or immediately after birth and do not cause hemodynamic problems. However, larger tumors can cause an obstruction, as in this case, and intervention is then needed."

Other differential diagnoses of rare neonatal cardiac tumors would include Burt-Hogg-Dube (FLCN gene) and Gorlin Syndrome (PTCH gene).

At birth, he was 39 weeks 3 days gestation, born via a repeat caesarian section; birth weight was $4.320 \mathrm{Kg} 97^{\text {th }}$ percentile, head circumference was $35.5 \mathrm{cms}$ on the $79^{\text {th }}$ percentile, and length was $53 \mathrm{CMS}$ on the $95^{\text {th }}$ percentile. Apgars at birth were 9 at 1 minute and 5 minutes.

The cardiac tumor was causing the boy to be hemodynamically unstable. The tumor was diagnosed as a cardiac rhabdomyoma by MRI and was impinging the mitral valve, causing mitral valve stenosis, left atrial hypertension, and pulmonary hypertension. Due to the position and severity of symptoms, the cardiac surgeons deemed it not prudent to remove the tumor. It was assessed and thought not to be a viable option due to post-surgical valvopathy. His brain MRI also supported the diagnosis of TSC with brain hamartomas. Genetic testing identified a known pathogenic heterozygous variant in TSC2 c.1832G>A, p. Arg611Gin.

It was thought the best option for him was the recommendation for evaluation for a cardiac transplant. Genetic testing included a SNP microarray which indicated a normal male and a negative methylation test for Beckwith -Wiedemann syndrome.
Due to the severity of the hemodynamic compromise, treatment was initiated at one week of age was with Everolimus (Afinitor®) $4.5 \mathrm{mg} / \mathrm{m}^{2} /$ day was started to reach a trough level of $5-15 \mathrm{ng} / \mathrm{ml}$. This level quickly exceeded the dose level needed and was supratherapeutic; this was decreased to $1 \mathrm{mg} / \mathrm{m}^{2} /$ day. One week later, the tumor was shrinking, and by one month of administration, the tumor was half the initial size seen at birth. See Images 1. And 2.

Update on this patient at two years of age. His last cardiac echo was negative for any tumor presence. He has not had a repeat of the MRI of the brain. This is currently pending, so I cannot comment on the effect on brain haemartomas. I can hypothesize that they will also have reduced in size as this was the initial use of Everolimus in TSC for the Subependymal giant cell astrocytomas (SEGA's) (2)

This patient had three eye tumors, 2 in the right eye and one in the left. Per the most recent ophthalmology review, they have not reduced, so there appears to be no improvement with the treatment.

Almost all fetuses with multiple cardiac rhabdomyomas will have TSC. They will often be the presenting feature (3)

Everolimus initiation has been shown to reduce cardiac tumor volume in this patient. A study is needed to ascertain if this treatment will need to be lifelong or if regression could be permanent. The EXIST-1 study supported the hypothesis that everolimus can safely reverse multisystem manifestations of TSC in a significant number of patients (4)

\section{"Everolimus initiation has been shown} to reduce cardiac tumor volume in this patient. A study is needed to ascertain if this treatment will need to be lifelong or if regression could be permanent."

Lessons learned and practical thoughts for the future:

1. Try to make a diagnosis ahead of time if a parent has TSC and a known pathogenic variant, amniocentesis for the TSC familial variant can be offered.

2. It is possible to consider the prenatal medication of the mother with an mTOR inhibitor during the pregnancy. Prenatal initiation of an mTOR inhibitor in TSC confirmed that as well

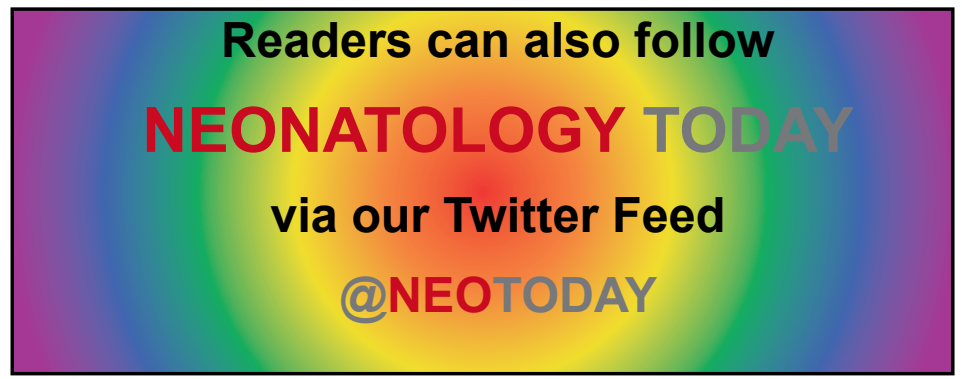




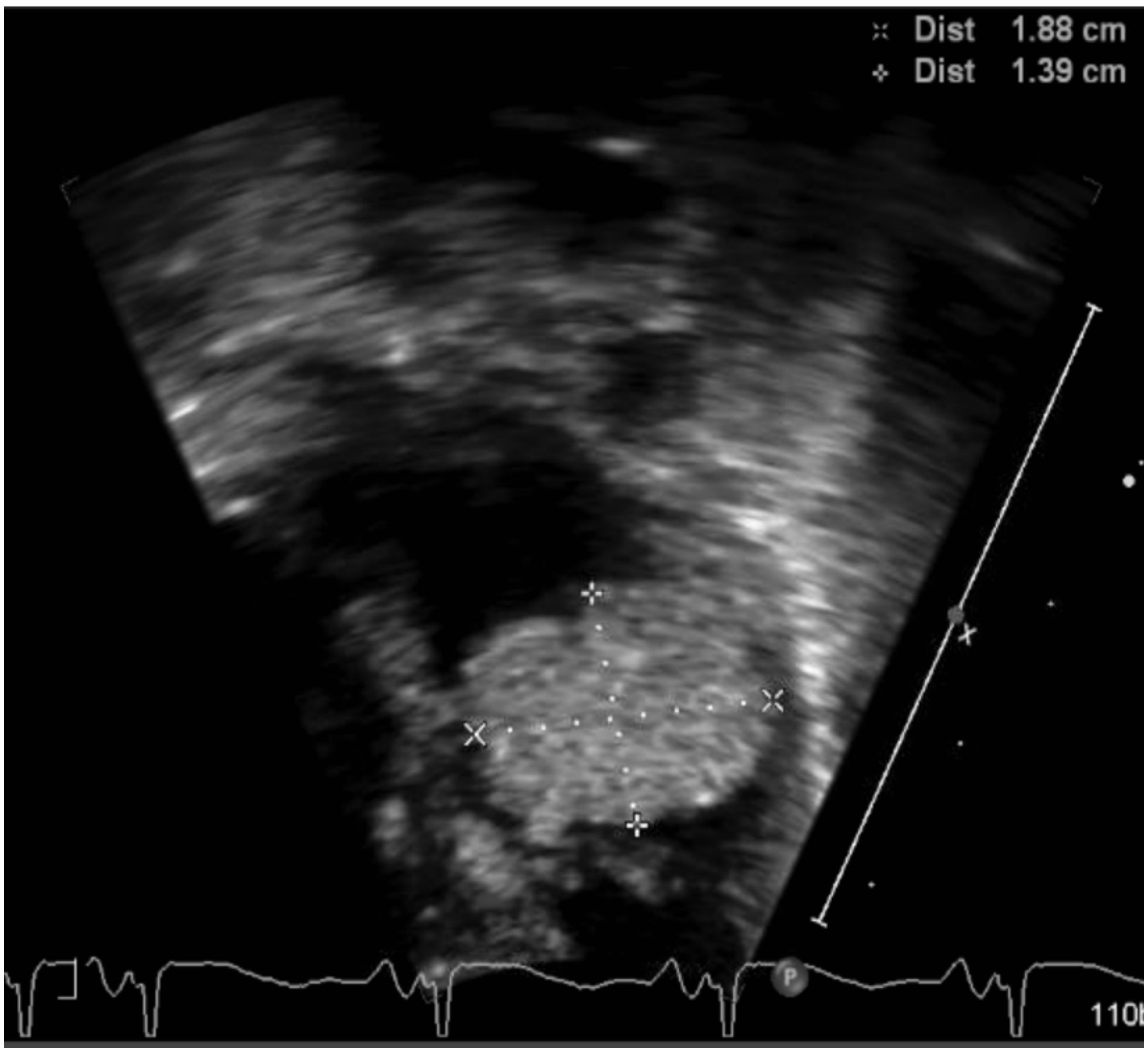

(1)Before mTOR inhibitor treatment

as a decrease in tumors, it may decrease the length of postnatal hospital stay and concurrent morbidities.

3. Anticipate the need for genetic testing and test rapidly if the infant is symptomatic to start treatment as soon as possible.

4. Everolimus has been shown to decrease cardiac tumor size in a TSC -related cardiac rhabdomyoma. It is feasible, but the dose-trough response has shown to be inconsistent in neonates.
5. Should we being prophylactic and treat all patients with TSC to prevent the multisystemic effects

\section{References:}

1. Sirolimus therapy forfetal cardiac rhabdomyoma in a pregnant woman with tuberous sclerosis Case Reports Obstet Gynecol Sci 2019 Jul;62(4): 280-284.doi: 10.5468/ogs.2019.62.4.280. Epub 2019 Jun 21. Hyea Park et al. PMID: 31338346

The only worldwide monthly publication
exclusively serving Pediatric and Adult
Cardiologists that focus on Congenital/
$\begin{aligned} & \text { CONGENITAL } \\ & \text { CARDIOLOGY } \\ & \text { SORAY }\end{aligned}$




\section{: Dist $0.822 \mathrm{~cm}$ \\ \& Dist $0.823 \mathrm{~cm}$}

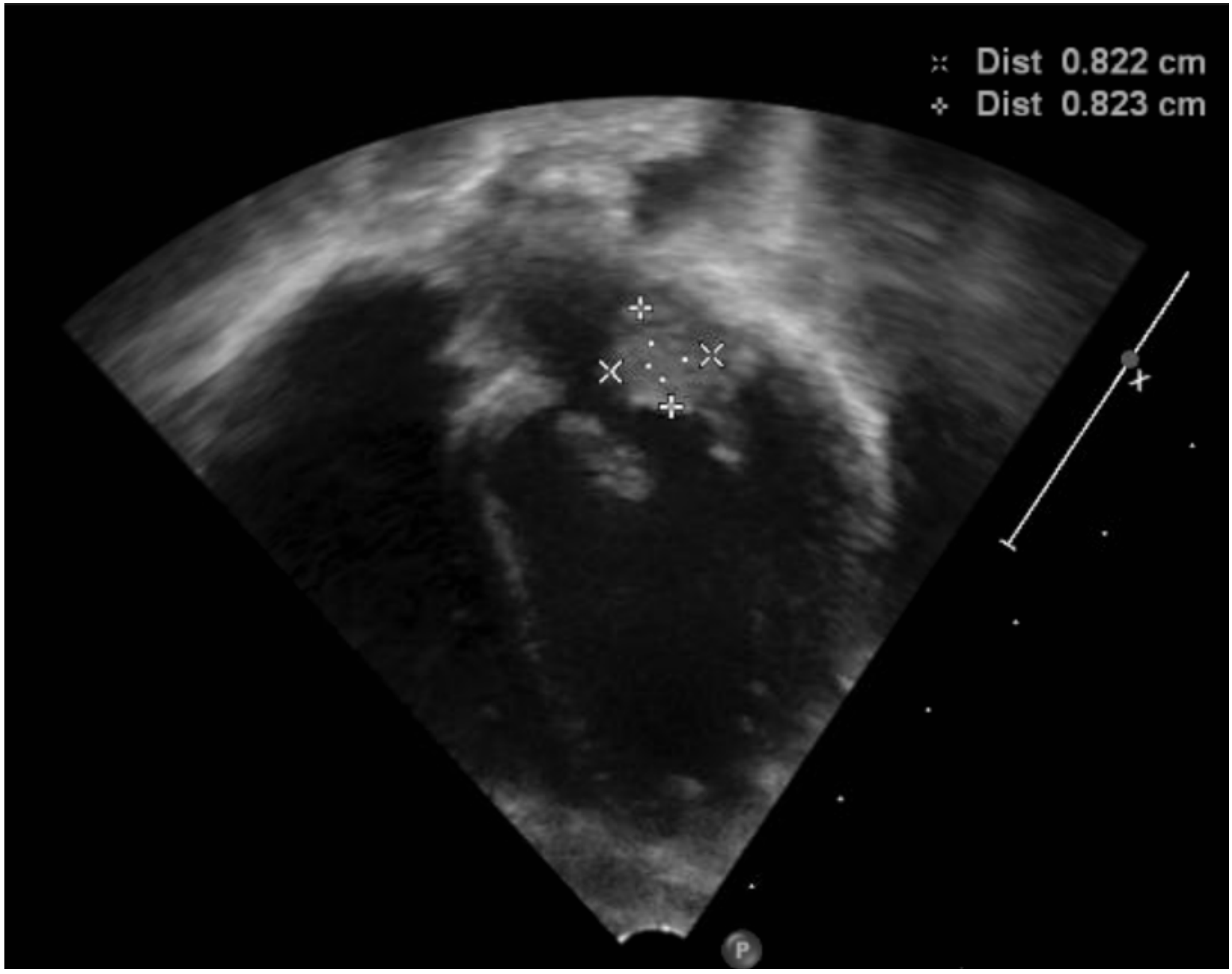

(2) One-month post-treatment with mTOR inhibitor

2. Everolimus for subependymal giant cell astrocytoma: 5-year final analysis. Franz DN, Agricola K, Mays M, Tudor C, Care MM, Holland-Bouley K, Berkowitz N, Miao S, Peyrard S, Krueger DA.Ann Neurol. 2015 Dec;78(6):929-38. doi: 10.1002/ana.24523. Epub 2015 Nov 9.PMID: 26381530

3. Dahdah, N Everolimus for the treatment of Tuberous Sclerosis Complex-Related Cardiac Rhabdomyomas in Pediatric Patients J Pediatr. 2017 Nov;190:21-26.e7. doi: 10.1016/j. jpeds.2017.06.076. Epub 2017 Sep 6.PMID: 28888564

4. Long-Term Use of Everolimus in Patients with Tuberous Sclerosis Complex: Final results from the EXIST-1 Study David N Franz et al. PMID: 27351628. Randomized Controlled Trial PLoS One.2016 Jun 28;11(6):e0158476. doi: 10.1371/journal.pone.0158476. eCollection 2016

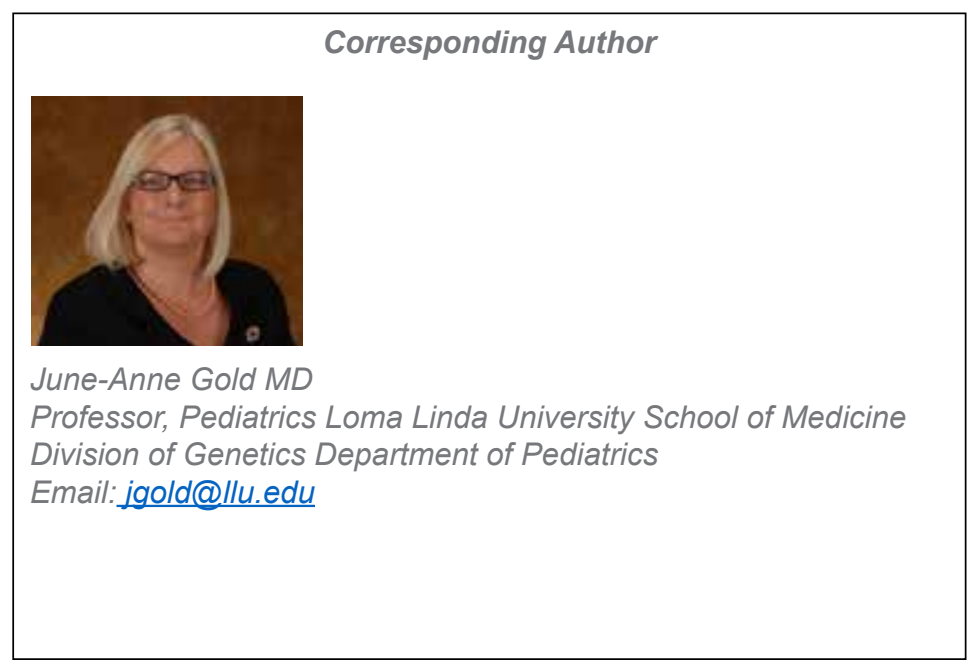

